\title{
Intermediate-high-risk pulmonary embolism: redefining it better with the support of the pulmonary embolism response teams approach
}

\author{
Tromboembolia pulmonar aguda de riesgo intermedio-alto: redefiniéndose mejor con el \\ apoyo de los equipos de respuesta rápida en tromboembolia pulmonar
}

\author{
Mateo Porres-Aguilar ${ }^{1 *}$, Javier E. Anaya-Ayala² and Luis E. Santos-Martínez ${ }^{3}$ \\ ${ }^{1}$ Hugo Castañeda M.D, P.A. Internal Medicine Clinic and Professional Associates; San Antonio, Texas; USA; ${ }^{2}$ Division of Vascular Surgery and \\ Endovascular Therapy, Department of Surgery, Instituto Nacional de Ciencias Médicas y Nutrición Salvador Zubirán, Mexico City, Mexico; \\ ${ }^{3}$ Departamento de Hipertensión Pulmonar y Corazón Derecho, Hospital de Cardiología, Centro Médico Nacional Siglo XXI, Instituto Mexicano del \\ Seguro Social, Mexico City, Mexico
}

Pulmonary embolism (PE) represents the third most common cause of cardiovascular death in western countries after acute coronary syndromes and stroke ${ }^{1-3}$. Recently, the 2014 European Society of Cardiology (ESC) guidelines further subdivided intermediate-risk PE into two subgroups: intermediate-low- and intermediate-high-risk PE, the latter, may portend worse prognosis with an estimated 30 -day mortality ranging from $5 \%$ to $25 \%$.

The current clinical trials focusing in reperfusion therapeutic strategies are still using their own arbitrary definition of the intermediate-risk (submassive) PE across studies (e.g., PEITHO, MOPETT, ULTIMA, and SEATTLE-II trials) perhaps, either underestimating or overestimating the disease. As previously emphasized and accurately pointed out by the ESC guidelines, not all the intermediate-risk patients are built the same or "fits all," do not have similar risk profile nor prognostic outcomes. Thus, we would like to propose a practical multimodality based, uniform, and homogeneous novel definition of intermediate-high-risk PE.

\section{Redefining intermediate-high-risk PE}

The redefinition of intermediate-high-risk PE encompasses a series of clinical findings (e.g., clinical severity scores that include tachycardia, tachypnea, hypoxemia, concomitant proximal deep vein thrombosis, previous comorbidities such as chronic pulmonary disease or heart failure, and high index for rapid clinical deterioration); electrocardiographic findings (e.g., new complete or incomplete right bundle branch block, and anteroseptal ST depression or elevation); echocardiographic and computed tomographic angiographic parameters (e.g., objective qualitative and quantitative signs of the right ventricular dysfunction); as well as the elevation of biomarkers suggesting myocardial injury (e.g., brain natriuretic peptide and troponin elevation) ${ }^{3,4}$. Table 1 integrates all the key aspects for such proposed novel definition of intermediate-high-risk PE.

\section{Pulmonary embolism response teams (PERT)}

We believe that through the implementation, adoption, and appropriate use of a designated hospital-based PERT, more rapid detection, risk stratification, formulation of a therapeutic strategy, and mobilization of specific resources (e.g., catheterization suite or the operating room for emergent pulmonary embolectomy) can be achieved for a given complex case of PE, and within

\footnotetext{
Correspondence:

*Mateo Porres-Aguilar

E-mail: mporres1980@gmail.com

Available online: 19-03-2019 Arch Cardiol Mex. 2019;89(1):55-57 www.archivoscardiologia.com 1405-9940 @ 2019 Instituto Nacional de Cardiología Ignacio Chávez. Publicado por Permanyer México SA de CV. Este es un artículo Open Access bajo la licencia CC BY-NC-ND (http://creativecommons.org/licenses/by-nc-nd/4.0/).
} 
Table 1. Proposal of important key points for a uniform, homogeneous, and standardized definition for intermediate-high-risk pulmonary embolism for further clinical trials and collection of data in research

— Integrated multimodality clinical risk stratification that includes clinical judgment, high index of suspicious for rapid deterioration (e.g., shock index, tachycardia, hypoxemia, and concomitant proximal deep vein thrombosis), severity grading scores such as the PESI > 106 points or the SPESI score $>1$ points.

- Objective evidence of RV dysfunction by echocardiographic or CTA criteria that include the following: Quantitative and qualitative echocardiographic findings such as RV/LV end-diastolic ratio $>1$ (seen at four-apical long-axis chamber views) or by CTA $>0.9, \mathrm{RV}$ end-diastolic diameter $>33 \mathrm{~mm}$, pulmonary arterial systolic pressure estimated $>30 \mathrm{mmHg}$, or tricuspid regurgitant jet velocity $>2.8 \mathrm{~m} / \mathrm{s}$.

- Significant electrocardiographic changes such as new complete or incomplete right bundle branch block, ST-segment depression, and/or significant T waves inversions in anteroseptal leads (V1-V4).

— Significant elevation of the biomarkers of myocardial injury: BNP $>100 \mathrm{pg} / \mathrm{ml}$ or N-terminal pro-BNP $>500 \mathrm{pg} / \mathrm{ml}$; troponin I $>0.4 \mathrm{ng} / \mathrm{ml}$, or troponin $\mathrm{T}>0.1 \mathrm{ng} / \mathrm{ml}$.

- $\mathrm{SI}>1(\mathrm{SI}=\mathrm{HR} / \mathrm{SBP})$ despite $\mathrm{SBP}>90 \mathrm{mmHg}$ (e.g., HR of $128 \mathrm{bpm}$ and SBP $105 \mathrm{mmHg}$ ), thus, predicting early clinical deterioration or hemodynamic collapse during the next $24-48 \mathrm{~h}$, requiring rescue reperfusion strategies (e.g., systemic thrombolysis or catheter-directed therapies).

PESI: pulmonary embolism severity index; sPESI: simplified PESI; RV: right ventricular; CTA: computed tomographic angiography; BNP: Brain natriuretic peptide; SI: shock index; HR: heart rate; SBP: systolic blood pressure

90 min after the activation or consultation of a PERT, formulated the best therapeutic modality by unanimous decision 5 . PERT leverages the exposure and expertise from various subspecialties across medicine while taking care of the most challenging and complex cases of $\mathrm{PE}$. These teams must require from the talent, experience, motivation, and close collaboration from multiple medical and surgical specialties such as emergency medicine physicians, acute hospitalists, intensivists/pulmonologists, interventional cardiologists and radiologists, cardiothoracic surgeons, vascular medicine, and hematologists with a focus in the evolving field of thrombosis ${ }^{5}$.

The first PERT was created in 2012 at the Massachusetts General Hospital in Boston. Since then, > 80 health-care institutions across the USA have adopted their PERT model ${ }^{6}$. The rationale and modus operandi from the PERT are based on the code heart and rapid response teams ${ }^{7}$. PERT facilitates expert knowledge exchange and skills among the multidisciplinary members, generating a consensus opinion to assess specific risks versus benefits for a certain therapeutic modality or intervention, especially in which lack of evidence-based medicine or clinical data exists ${ }^{5-7}$.

PERT members must be clinicians with a strong motivation, mutual interest, dedication, and full commitment to the care of PE, since these elements are keyed for the success of a given PERT.
We strongly believe that with the setup, initiation and worldwide dissemination of PERT will create the following benefits:

1. Provide fast, efficient, organized, individualized, and best evidence-based care in complex case of PE.

2. Provide collection and interpretation of database, thus, generating updated medical literature with publications in regards best care in PE.

3. Generate and perform high-quality care core measures in acute cardiovascular and pulmonary care.

4. Coordinate patient care with great collaborative team effort across multiple specialties, leveraging them to bring their experience and special skills and knowledge all together.

5. Further areas of clinical research in the field of venous thromboembolism.

6. A vehicle and dissemination of education not only to our physicians but also to our patients.

7. Represent soon, a shifting paradigm and new standard of care in the world of acute PE.

PERT represents a novel process, approach, and strategy, which had emerged to advance, individualized in a case-by-case basis, and personalized therapeutic modalities, especially for intermediate-high and high-risk PE patients, positively impacting quality of life and survival.

\section{Conflicts of interest}

The authors declare no conflicts of interest. 


\section{Ethical disclosures}

Protection of human and animal subjects. The authors declare that no experiments were performed on humans or animals for this study.

Confidentiality of data. The authors declare that they have followed the protocols of their work center on the publication of patient data.

Right to privacy and informed consent. The authors declare that no patient data appear in this article.

\section{References}

1. Jaff M, McMurtry M, Archer S, et al. Management of massive and submassive pulmonary embolism, iliofemoral deep vein thrombosis, and chronic thromboembolic pulmonary hypertension: a scientific statement from the American heart association (AHA). Circulation. 2011; 123:1788-830.

2. Konstantinides SV, Torbicki A, Agnelli G, et al. 2014 ESC guidelines on the diagnosis and management of acute pulmonary embolism. Eur Heart J. 2014;35:3033-69, 3069a-3069k.

3. Teleb M, Porres-Aguilar M, Anaya-Ayala JE, et al. Potential role of systemic thrombolysis in submassive intermediate risk acute pulmonary embolism: review and future perspectives. Ther Adv Cardiovasc Dis. 2016;10:103-110.

4. Porres-Muñoz M, Porres-Aguilar M. Intermediate-high-risk pulmonary embolism: standardizing definition and optimizing therapeutic strategies. Am J Med. 2017;130:e233.

5. Porres-Aguilar M, Anaya-Ayala JE, Heresi GA, Rivera-Lebron B. Pulmonary embolism response teams: a novel approach for the care of complex patients with pulmonary embolism. Clin Appl Thromb Hemost. 2018 Doi: 10.1177/1076029618812954. [Epub ahead of print].

6. Available from: https://www.pertconsortium.org. [Last accessed on 2018 Jun 19].

7. Holmes DR, Rich JB, Zogbhi WA, et al. The heart team of cardiovascular care. J Am Coll Cardiol. 2013;63:903-7. 